\title{
Grasping Using Magnetically-Actuated Tentacle Catheter: A Proof-Of-Concept Study
}

\author{
Jakub Sikorski, Etienne S.A.A.M Rutting, and Sarthak Misra
}

\begin{abstract}
The use of magnetically-actuated catheters has the potential to facilitate minimally-invasive surgical procedures. In this work we present a magnetically-actuated tentacle catheter for object manipulation in remote areas of human body. The catheter employs a bio-inspired technique of underactuated grasping. The whole body of the catheter loops around the target object, providing form closure necessary for manipulation. The catheter employs a permanent magnet to steer the position of its tip, and an electromagnetic coil to strengthen the maximum force applicable to the target object. We test the catheter in a series of proof-of-concept experiments. In a thermal study, we show that within the operational conditions, the heat dissipated by the coil allows for safe operation of tentacle catheter within human body. Subsequently, we characterise the maximum force available for manipulation using force sensor. The tentacle catheter can apply forces up to $0.1 \mathrm{~N}$, which is in accordance with finite-element simulation. This force is sufficient for object manipulation in surgical tasks, such as biopsy. Finally, we demonstrate the operation of the tentacle catheter in a task involving manipulation of porcine tissue.
\end{abstract}

\section{INTRODUCTION}

In the last few years, research in continuum robotics has developed new classes of tools for minimally-invasive surgical (MIS) procedures [1]. Among these tools, automated catheters exhibit potential of becoming versatile robotic manipulators, capable of performing various surgical tasks inside the human body. Standard, manually-operated catheters are mostly used for a small number of MIS procedures within endovascular surgery. The applications of standard catheters are limited mostly due to lack of precision, resulting in time-consuming navigation, which is challenging even for experienced clinicians. By employing automated steering techniques, the precision of catheters can be improved, making them easier to operate. This would not only improve the outcome of current procedures, but also extend the number of MIS procedures which can be performed using catheters [2].

The versatility of catheters comes from their favourable mechanical properties. They comprise of sleek, soft polymeric tubes with inherent compliance, which facilitates safe operation within the human body. Small size of the catheters enables them to access difficult locations with minimal trauma. Steering of larger catheters is usually performed

The authors are affiliated with Surgical Robotics Laboratory, Departmen of Biomechanical Engineering, University of Twente, 7500 AE Enschede, The Netherlands. S. Misra is also affiliated with the Department of Biomedical Engineering, University of Groningen and University Medical Centre Groningen, 9713 GZ Groningen, The Netherlands.

This research has received funding from the European Research Council (ERC) under the European Unions Horizon 2020 Research and Innovation programme (Grant Agreement \#638428 - project ROBOTAR).

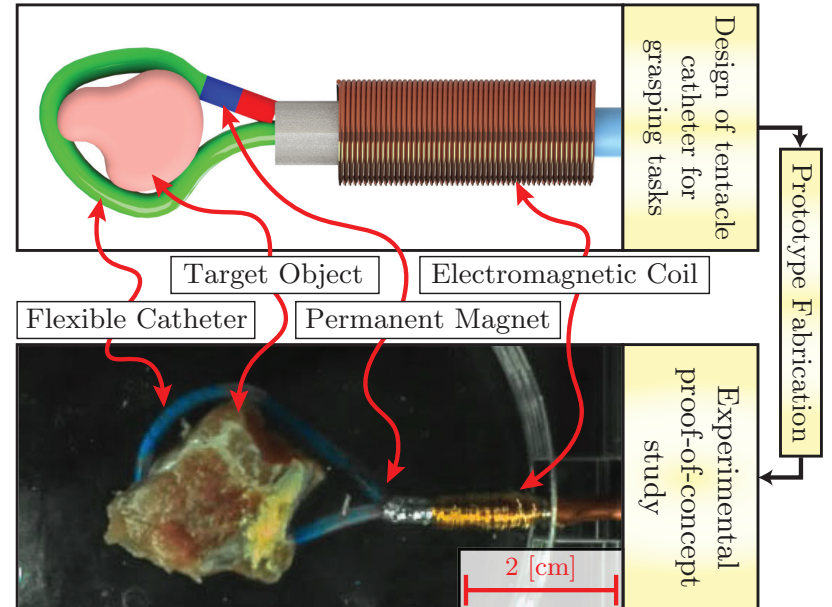

Fig. 1. In this study we propose a catheter for execution of grasping tasks for minimally-invasive surgical procedures. The catheter employs and under-actuated grasping technique, looping its whole body around the target object. For that purpose, the catheter is steered using magnetic actuation enabled by a permanent magnet at its tip. Furthermore, an electromagnetic grasp, allowing for reliable object manipulation. This concept, bio-inspired by tentacle grasping has been selected during the design phase, due to suitability for operation within human body, as well as for the compatibility with pre-existing magnetic systems. A prototype of the tentacle catheter has been fabricated and tested during the series of proof-of-concept experiments. Among other tests, we demonstrate that the tentacle catheter is capable of grasping and manipulating porcine tissue during a pick-and-place tasks.

using tendons [3]. However, friction in tendon-driven instruments leads to safety concerns and limits their scalability [4]. Furthermore, the tendons hinder compliance matching between the catheter and the human tissue, decreasing the benefits of using flexible catheters [5].

Magnetic actuation has been investigated as a viable solution to these problems [6]. Several studies have already demonstrated steering of magnetically-actuated catheters. Nevertheless, the principal emphasis therein is placed on establishing theoretical foundation of catheter mechanics and control [7]-[10]. Despite being a remarkable milestone, these studies seek to improve the performance of catheters for standard MIS procedures. A few other studies suggested application of magnetic catheters for eye surgery, but their focus does not go beyond the standard task involving developing a point contact between the catheter tip and the tissue [11], [12].

Current literature certainly does not exhaust the opportunities offered by magnetic catheters. One of the tasks, which in the future can be realised by catheters is grasping, i.e., achieving a stable physical contact and force transmission between the manipulator and target object. It is required for object manipulation in various MIS procedures such as biopsies, keyhole surgery and targeted drug delivery. The grasping tasks in those procedures are usually realised by 


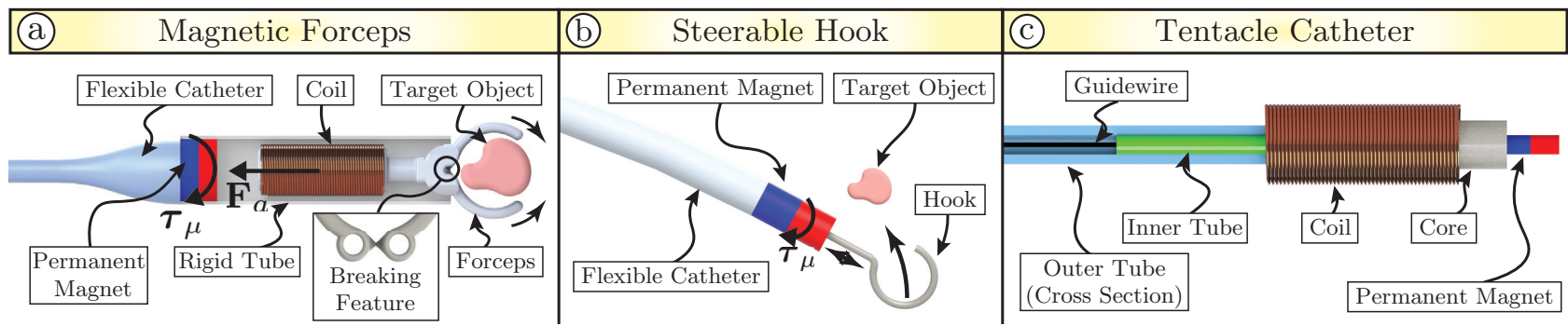

Fig. 2. The results of conceptual design study on grasping catheters. The final solution is chosen from three generated concepts. The two initial concepts involve magnetically actuated forceps (a) and hook (b). The final design (c) addresses the limitations of the discarded concepts. It employs a catheter comprising of two concentric tubes. The inner tube has a permanent magnet at its tip. The grasping is realised by ejecting the inner tube using a guidewire, and steering it using magnetic torque $\left(\boldsymbol{\tau}_{\mu}\right)$ to wrap around a target object. The grasping is strengthened by using an electromagnetic coil attached to the outer tube. Running the current inside the coil results in generation of large magnetic attraction force $\left(\mathbf{F}_{a}\right)$, attracting the tip magnet.

mechanically-complex jaw-like forceps [13]. These forceps are hard to miniaturize and actuate remotely, therefore their dexterity within human body is limited [14].

In this work we design and test a magnetically-actuated tentacle catheter for grasping tasks inside the human body. This catheter employs bio-inspired under-actuated grasping technique. This technique has been demonstrated in larger, industrial continuum manipulators, but has not yet been applied for medical procedures [15]. Due to favourable distribution of the contact force, continuum manipulators employing under-actuated grasping are considered safer for object manipulation than classical forceps [5]. Moreover, their simple design allow for successful miniaturization and use in MIS procedures.

The tentacle catheter presented in this study employs a permanent magnet at its flexible tip. The primary function of the magnet involves steering of the catheter into the desired location and looping it around a target object, using the interaction with external magnetic fields. However, due to high compliance of the catheter, the force required for manipulation of the object deforms the catheter tip, preventing successful grasps. As a remedy to that problem, we incorporate an electromagnetic coil into the body of the catheter. With the tip of the catheter looped around the target object, the coil is used to exert a large attraction force on the permanent magnet. This force strengthens the grasp and allows for object manipulation. We test our design experimentally, using BigMag for magnetic actuation [17]. We employ a force sensor to quantify the strength of the grasp and compare the results with a finite element simulation. Finally, we demonstrate the tentacle catheter performing a simple pick-and-place task using biological material.

The rest of this paper is structured as follows. Section II introduces and analyses three proposed conceptual designs for grasping catheters, and presents the details of the tentacle catheter employing under-actuated grasping. Section III shows the results of a proof-of-concept study demonstrating the feasibility of the tentacle catheter. Finally, Section IV summarises the paper, giving recommendations for further study.

\section{CATHETER AS A GRASPING TOOL - CONCEPTUAL DESIGN}

To authors best knowledge, the idea of realising grasping tasks within human body using (magnetically-actuated) catheters has not yet been discussed in the literature. This necessitates starting the investigation with a design process, in which possible grasping strategies are generated and translated into novel concepts of catheters. This section describes that process, proposing and analysing three concepts of grasping catheters. The concepts are assessed using a set of design requirements. Based on these requirements, we show that the catheter employing under-actuated grasping shows distinct advantages in terms of its versatility, applicability and miniaturization capabilities. Thus, in this section we give the details of the proposed under-actuated grasping strategy, chosen to be verified further in the paper with a set of proofof-concept studies.

\section{A. Design Requirements and Preliminary Concepts}

This study aims at development of a magnetically-actuated catheter with a potential of performing grasping tasks within remote locations inside the human body. We begin by establishing a set of requirements for such a device. Using those requirements, three concepts for magnetically-actuated grasping catheters are generated during the design phase (Figure 2). The concepts are analysed and assessed for their conformity with the design requirements. Table I lists the requirements and summarises the results of our analysis, leading to the selection of the final design.

The first concept aims at miniaturizing the standard, robotic forceps using magnetic actuation (Figure 2.(a)). The forceps are located at the tip of the catheter in a hollow, rigid tube extending from the flexible body of the catheter. A permanent magnet located at the end of the tube is used both in catheter steering and grasping. The forceps are 3D printed as a single part, held open by a small portion of material called breaking feature. The forceps are fitted with electromagnetic coil. When delivered to the location of the target object, the electromagnetic coil is activated. This creates a magnetic attraction force $\left(\mathbf{F}_{a} \in \mathbb{R}^{3}\right)$ pulling the forceps towards the magnet. This force destroys the breaking feature, allowing the forceps to grasp the target object.

After careful analysis of the design requirements, the forceps are considered unsuitable for the target application for a number of reasons. The complex mechanical structure of the forceps is difficult to manufacture using rapid-prototyping techniques. Since a breaking feature is used, the forceps are not reusable. After a grasping attempt, the catheter loses its functionality and needs to be extracted from the body. Furthermore, the resulting end-effector is considered too stiff to allow for safe operation within the body. 
TABLE I

DESIGN REQUIREMENTS FOR GRASPING CATHETER

\begin{tabular}{|c|c|c|c|c|}
\hline \multirow[b]{2}{*}{ Requirement } & \multirow[b]{2}{*}{ Motivation } & \multicolumn{3}{|c|}{ Catheter Concepts } \\
\hline & & $\begin{array}{l}\text { Magnetic } \\
\text { Forceps }\end{array}$ & $\begin{array}{l}\text { Steerable } \\
\text { Hook }\end{array}$ & $\begin{array}{l}\text { Tentacle } \\
\text { Catheter }\end{array}$ \\
\hline $\begin{array}{l}\text { The catheter is capable of grasping objects } \\
\text { within human body. }\end{array}$ & $\begin{array}{l}\text { Primary function of the catheter. Therefore, also the } \\
\text { main design requirement. }\end{array}$ & $\sqrt{ }$ & $\checkmark$ & $\checkmark$ \\
\hline $\begin{array}{l}\text { The catheter has sleek shape and is primarily } \\
\text { made of flexible materials. }\end{array}$ & $\begin{array}{l}\text { Improves safety and dexterity of the device inside the } \\
\text { cavities of human body. }\end{array}$ & $x$ & $\checkmark$ & $\checkmark$ \\
\hline The catheter has a simple and robust structure. & $\begin{array}{l}\text { Limits the number of possible failure scenarios, } \\
\text { contributing to safety of the patient. }\end{array}$ & $x$ & $\sqrt{ }$ & $\sqrt{ }$ \\
\hline $\begin{array}{l}\text { The concept has a potential for miniaturization } \\
\text { using currently avalable fabrication techniques. }\end{array}$ & $\begin{array}{l}\text { Increases the number of potential applications within } \\
\text { minimially-invasive surgery. }\end{array}$ & $x$ & $\sqrt{ }$ & $\sqrt{ }$ \\
\hline $\begin{array}{l}\text { The catheter is reusable after performing a } \\
\text { grasping task. }\end{array}$ & $\begin{array}{l}\text { Allows for manipulation of multiple objects during a } \\
\text { single procedure. }\end{array}$ & $x$ & $x$ & $\checkmark$ \\
\hline $\begin{array}{l}\text { The grasping technique is compatible with } \\
\text { pre-existing devices for magnetic manipulation. }\end{array}$ & $\begin{array}{l}\text { Ensures the concept is realistic and can be realised } \\
\text { within the current state-of-the-art technology. }\end{array}$ & $x$ & $x$ & $\sqrt{ }$ \\
\hline
\end{tabular}

In an attempt to address those challenges a second concept is devised, in which the forceps are substituted with a hook (Figure 2.(b)). Having a much simpler mechanical structure, a hook offers fewer failure scenarios, and can be successfully fabricated at different scales. Albeit appealing, this concept revealed another set of problems inherent to grasping using catheters. For successful grasp, both the forceps and the hook need to approach the target object at a particular orientation. This is enabled only if the manipulator allows for $6 \mathrm{DoF}$ control of the end-effector pose. This is challenging to realise due to limitations of magnetic actuation, and has not yet been demonstrated in the literature [9].

Ultimately, we propose the catheter realising the underactuated grasping technique [15]. This technique is inspired by biological structures like vines, tentacles or elephant trunks. The device is sleek and compliant; its structure is simple and shows potential for miniaturization. The underactuated grasping technique is versatile, allowing the device to grasp targets of different sizes and shapes. Furthermore, the grasping using this technique does not require precise pose control of catheter tip. Thus, the tentacle catheter is compatible with the existing systems for magnetic actuation. Therefore this design fulfils all the requirements presented in Table I.

\section{B. Details of Tentacle Catheter}

The proposed tentacle catheter (Figure 2.(c)) comprises of two concentric, compliant tubes. The elastic modulus of the outer tube is comparable to standard medical catheter (300 $\mathrm{MPa}$ ), whereas the modulus of the inner tube is an order of magnitude lower (50 MPa). The outer tube spans the entire length of the device, from the point of insertion to the tip of the catheter. The inner tube is short, and at its proximal end it connects to a guidewire used to control its position inside the outer tube.

The grasping strategy is employed in tentacle catheter is presented in Figure 3. A permanent magnet, represented by its magnetic dipole moment $\left(\boldsymbol{\mu} \in \mathbb{R}^{3}\right)$, is attached at the tip of the inner tube. Exposed to external magnetic field $\left(\mathbf{B} \in \mathbb{R}^{3}\right)$, the catheter experiences a wrench $\left(\mathbf{W} \in \mathbb{R}^{6}\right)$ comprising of magnetic force $\left(\mathbf{F}_{\mu} \in \mathbb{R}^{3}\right)$ and magnetic torque $\left(\boldsymbol{\tau}_{\mu} \in \mathbb{R}^{3}\right)$ as follows:

$$
\mathbf{W}=\left[\begin{array}{c}
\mathbf{F}_{\mu} \\
\boldsymbol{\tau}_{\mu}
\end{array}\right]=\left[\begin{array}{c}
\nabla(\boldsymbol{\mu} \cdot \mathbf{B}) \\
\boldsymbol{\mu} \times \mathbf{B}
\end{array}\right]
$$

In tentacle catheter, this wrench has two main applications, depending on the phase of the task. During the navigation through the body to the target site, the inner tube is fully retracted. Hence, the wrench $(\mathbf{W})$ is transmitted directly to outer tube. This allows for the navigation of the catheter to the desired pose within the target site in a manner similar to [7]. When the catheter reaches the target site, the inner tube is extended using the guidewire. The wrench $(\mathbf{W})$ is then used to bend the inner tube around the target object, realising the under-actuated grasp. Since the compliance of the inner tube is much lower than the outer one, this bending can be realised with much lower magnitude of $\mathbf{W}$.

Once the catheter is wrapped around the target object, the contact between the object and the body of the manipulator provides planar form closure [15]. However, due to high compliance of the inner tube, the wrench $(\mathbf{W})$ itself may not be sufficient to maintain stable grasp, especially if the manipulation of the object requires overcoming external forces. Thus, we modify the standard under-actuated grasping strategy to alleviate that problem. We locate an electromagnetic coil at the end of the outer tube. Once the inner tube of the catheter is bent around the target object, the coil is activated to attract the tip magnet with a magnetic force $\left(\mathbf{F}_{a} \in \mathbb{R}^{3}\right)$, forming a loop around a target object. This loop can be tightened using the guidewire to form a stable grasp. This grasp is strengthened by $\mathbf{F}_{a}$, which deteremines

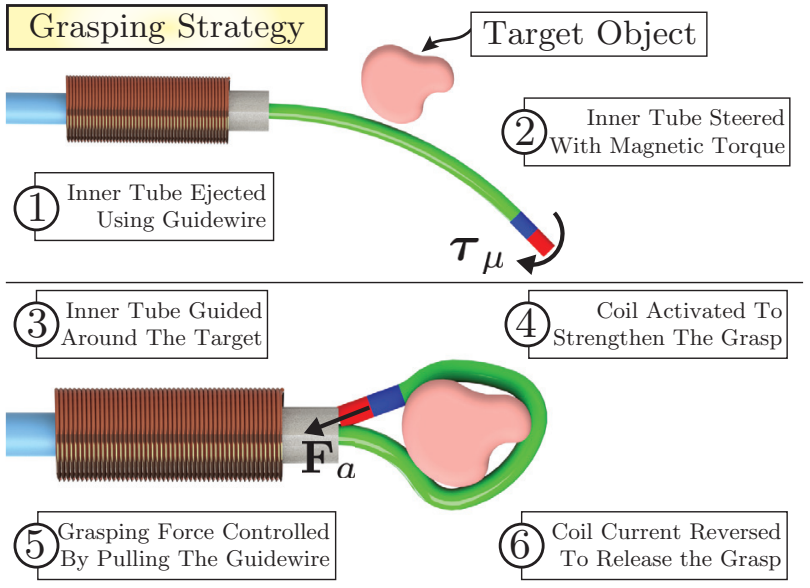

Fig. 3. The steps (1) to (6) present the proposed grasping strategy. The catheter is navigated towards the target site using magnetic actuation. The inner tube of the catheter is ejected and steered around a target object, using magnetic torque $\left(\boldsymbol{\tau}_{\mu}\right)$. The electromagnetic coil located on the outer tube is activated to attract the tip magnet using the force $\left(\mathbf{F}_{a}\right)$ forming a stable loop around the target object. By pulling the guidewire, the loop is tightened, grasping the target object. The magnitude of $\mathbf{F}_{a}$ determines the maximum possible grasping force. 


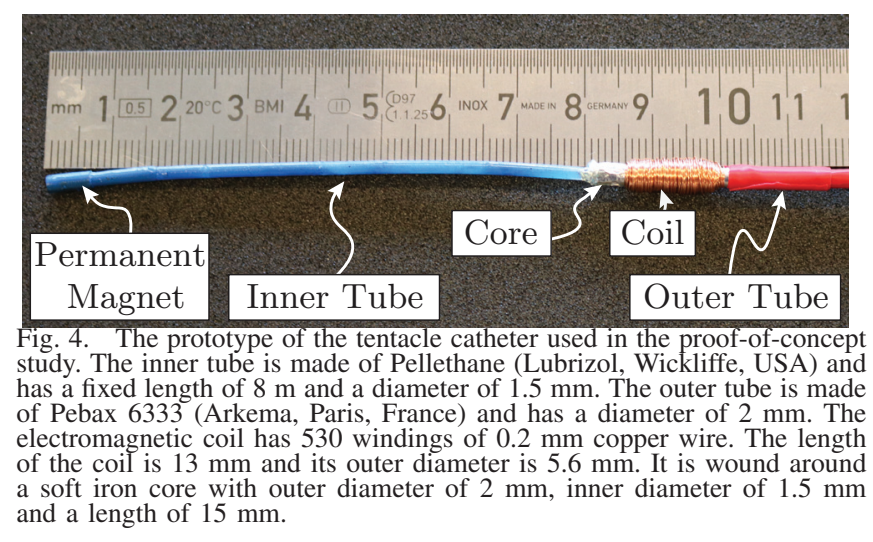

therefore the maximum force, which can be transmitted to the target object during the manipulation task.

\section{Tentacle CATHEter - EXPerimental PROOF-OF-CONCEPT}

After selecting the tentacle catheter as the final concept for grasping tasks, we fabricate it and demonstrate its capabilities in a series of proof-of-concept studies. The prototype of the catheter used for that purpose is presented in Figure 4. The mechanism for ejecting/retracting the inner tube using a guidewire (Figure 3, steps (1) and (5)) is not realised in our prototype. The inner tube is kept ejected at a constant length of $8 \mathrm{~mm}$. Such a catheter can successfully loop around objects with circumferences smaller than this value. The resulting grasp does not fully constrain the relative motion between the target and the manipulator body. Nevertheless, the resulting form closure allows for planar manipulations, and thus it is considered sufficient for the proof-of-concept experiments.

Three studies have been performed to test the suitability of tentacle catheter for grasping tasks. Since the heat dissipated due to resistance of the electromagnetic coil can damage the tissues of human body, we perform a thermal study to demonstrate the safety of the catheter within the desired range of currents. Consequently we test the tentacle catheter in two experiments. We characterise the maximum strength of the grasp experimentally using a force sensor, and compare it with a finite element (FE) simulation. Finally, we demonstrate that the tentacle catheter can be used for object manipulation by performing grasping task using porcine tissue.

\section{A. Thermal Safety Study}

The grasping technique proposed for tentacle catheter involves using an electromagnetic coil to increase the maximum manipulation force. The coil is operated using small direct currents (maximum of $1.25 \mathrm{~A}$ at a voltage of 5.3 V), which pose no threat to human tissues. However, due to resistance of the windings, the coil dissipates substantial amount of heat. In contrast to the current, this heat can be damaging to the tissues surrounding the catheter. Therefore, demonstrating the thermal safety of the tentacle catheter is an important part of the proof-of-concept study.

The experimental setup used for studying thermal safety is presented in Figure 5. The prototype of the tentacle catheter is located in the tank of water kept at a room temperature. A
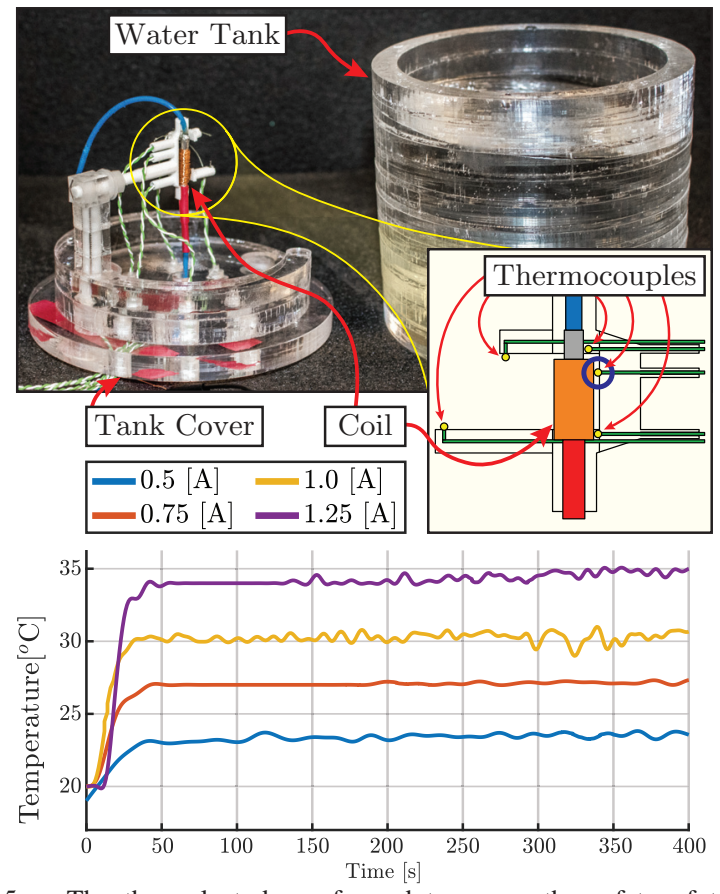

Fig. 5. The thermal study performed to assess the safety of tentacle catheter. The prototype of the tentacle catheter shown in Figure 4 is held in a tank of water, which is initially at room temperature. 5 Z2-T-1M thermocouples (LABFACILITY, Bognor Regis, The UK) are used to record thermocouples (LABFACILITY, Bognor Regis, The UK) are used to record
the temperature at different points around the coil of the catheter. Constant current of fixed magnitudes is run through the coil, which causes the coil temperature increase due to resistive heating. The temperature increase at four different values of current is recorded. The graph presents the readings from one thermocouple closest to the body of the coil (marked with purple circle in the figure). The temperature increase recorded further away from the coil was negligible in all cases.

constant current is run through the coil. The heat dissipated by the coil increases the temperature of the environment. A set of 5 Z2-T-1M thermocouples (LABFACILITY, Bognor Regis, UK) is used to record the temperature at different locations around the coil. After approximately 30 seconds, the coil reaches thermal equilibrium. We observe that for the thermocouple located the closest to the coil, the maximum temperature difference at that equilibrium is $15{ }^{\circ} \mathrm{C}$ for the current of $1.25 \mathrm{~A}$. The thermocouples located more distant from the coil recorded negligible temperature differences at all current values.

For the current of $0.75 \mathrm{~A}$ the coil temperature increases by $7{ }^{\circ} \mathrm{C}$, which has been shown to cause negligible tissue damage in an in vivo study [16]. This allows us to conclude that, if operated at $0.75 \mathrm{~A}$, the prototype of tentacle catheter can be considered suitable for grasping tasks within human body. Therefore, we decide to use this current value for grasping task further in the paper.

\section{B. Estimation of Maximum Grasping Force}

Manipulation tasks require the tentacle catheter to apply force to the target object. The inherent compliance of the catheter imposes limits on the magnitude of that force. In our grasping strategy (Figure 3), we proposed using an electromagnetic coil to increase the maximum force, which can be applied to the object without deforming the catheter and breaking the contact. In this section we characterise the maximum applicable force, Furthermore, we investigate its relationship with the attraction force $\left(\mathbf{F}_{a}\right)$ between the tip 

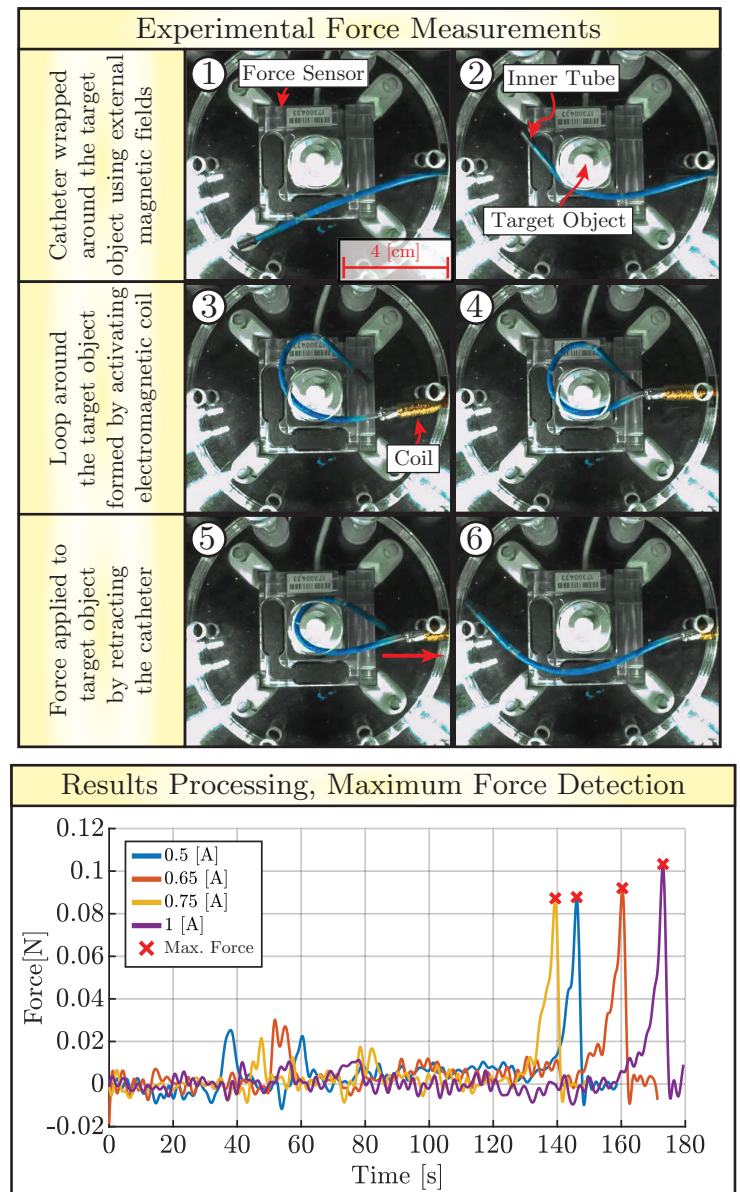

Fig. 6. Experiment used to determine the maximum force applicable with tentacle catheter. The catheter is inserted into the workspace of BigMag array using an automated inserter (1). External magnetic field is used to wrap the catheter around a circular target object attached to K3D40 force sensor (Meßsysteme, Hennigsdorf, Germany) (2) - (3). After the activation of the coil, the catheter forms a stable loop around the object (4). Following this, the catheter is slowly retracted, applying force to the target object (5). If the force applied to the object is too large, the loop breaks and the catheter detaches from the object (6). The experiment is repeated for 4 values of coil currents. The maximum forces applicable with the tentacle catheter are within the range of $0.08-0.1 \mathrm{~N}$. Please refer to the video in the supplementary material for demonstration of this experiment.

magnet and the electromagnetic coil using FE simulation.

The force characterization experiment for the tentacle catheter is presented in Figure 6. The catheter is placed inside the workspace of BigMag - an array of mobile electromagnets [17]. Magnetic field generated by BigMag is used to wrap the catheter around a target object connected to K3D40 force sensor (Meßsysteme, Hennigsdorf, Germany). Once this task is finished, we activate the coil to form a loop around the target object. Subsequently, the catheter is retracted using an automated inserter. This results in a force being applied to the target object. This force is measured by the force sensor. Once the force exceeds a certain value, it breaks the connection between the tip magnet and the coil, releasing the target object. We determine this maximum applicable force by inspecting the measurements from the force sensor. The experiment is repeated for four values of coil current. All the results are comparable, and lie within the range of $0.08-0.10 \mathrm{~N}$. This force allows for manipulation against gravity of objects several orders of magnitudes heavier than the standard tissue samples extracted for dur-
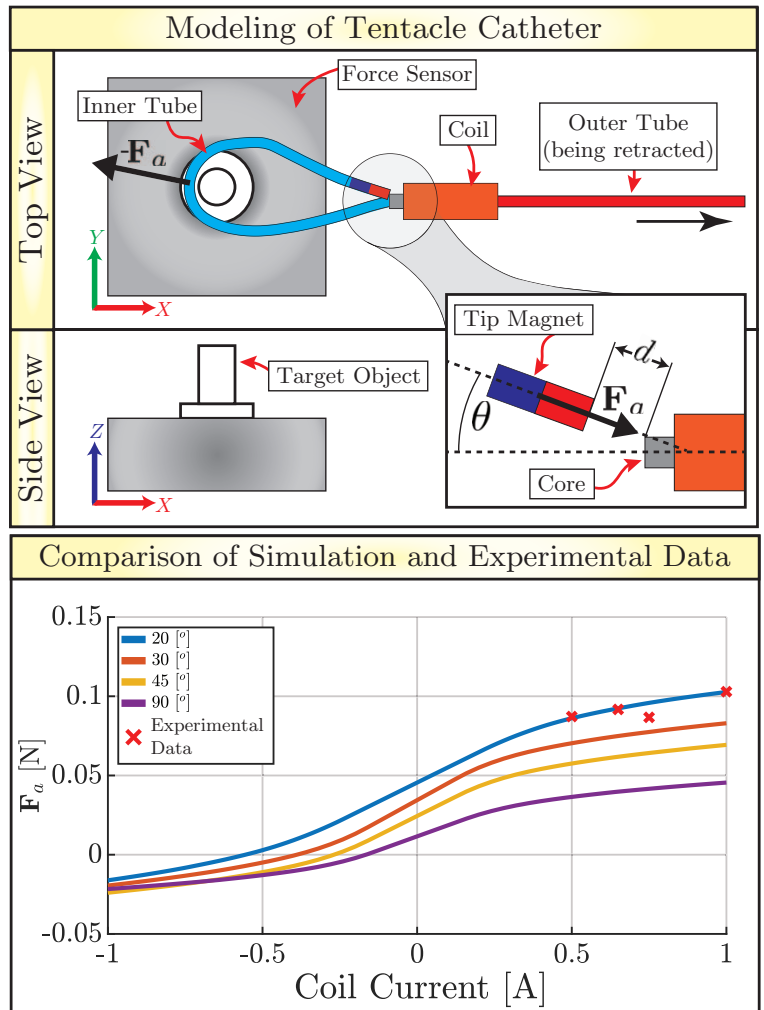

Fig. 7. The relation between the attraction force $\left(\mathbf{F}_{a}\right)$ and the maximum force applicable by the tentacle catheter is investigated using finite element simulation. COMSOL 5.2 (Comsol Inc., Burlington, USA) is used to model the attraction between a permanent magnet located at the tip of the catheter and the electromagnetic coil. The assumed distance $\left(d \in \mathbb{R}^{+}\right)$between the magnet and the coil is $0.15 \mathrm{~mm}$, which accounts for thickness of nonmagnetic coating. The attraction force $\left(\mathbf{F}_{a}\right)$ is computed for 4 different values of angle $(\theta \in \mathbb{S})$ between the magnet and the coil. The values of the component of $\mathbf{F}_{a}$ oriented towards the coil are compared against the maximum applicable forces (red crosses) determined experimentally (Figure 6). The results show that the maximum force which can be applied using tentacle catheter is directly related to the magnitude of the attraction force $\left(\mathbf{F}_{a}\right)$ between the tip magnet and the electromagnetic coil. Furthermore, the predicted force value from the proposed attraction model closely matches experimental observations.

ing biopsies [18]. Therefore, we consider tentacle catheter to be clinically-relevant.

The dependence of the maximum applicable manipulation force on the attraction force $\left(\mathbf{F}_{a} \in \mathbb{R}^{3}\right)$ between the tip magnet and the electromagnetic coil is verified using finite element simulations (Figure 7). The model used for the simulation involves a coil and a permanent magnet kept at a distance $\left(d=0.15 \mathrm{~mm} \in \mathbb{R}^{+}\right)$from one another. The magnet is kept at an angle $(\theta \in \mathbb{S})$ from the long axis of the coil. COMSOL 5.2 (Comsol Inc., Burlington, USA) is used to compute the value of $\left(\mathbf{F}_{a}\right)$ at a range of coil currents and angles $(\theta)$.

We assume that only the component of $\mathbf{F}_{a}$ along the axis of magnet dipole moment $(\boldsymbol{\mu})$ plays role in stabilising the loop. The value of that component is compared against the experimental results. We observe close correspondence of the forces measured in Figure 6 and the computed magnitudes of attraction force. Furthermore, the simulation predicts that approximately $0.5 \mathrm{~A}$ of current in the reverse direction is needed to break the connection between the tip magnet and the coil. This value matches with experimental observations. 


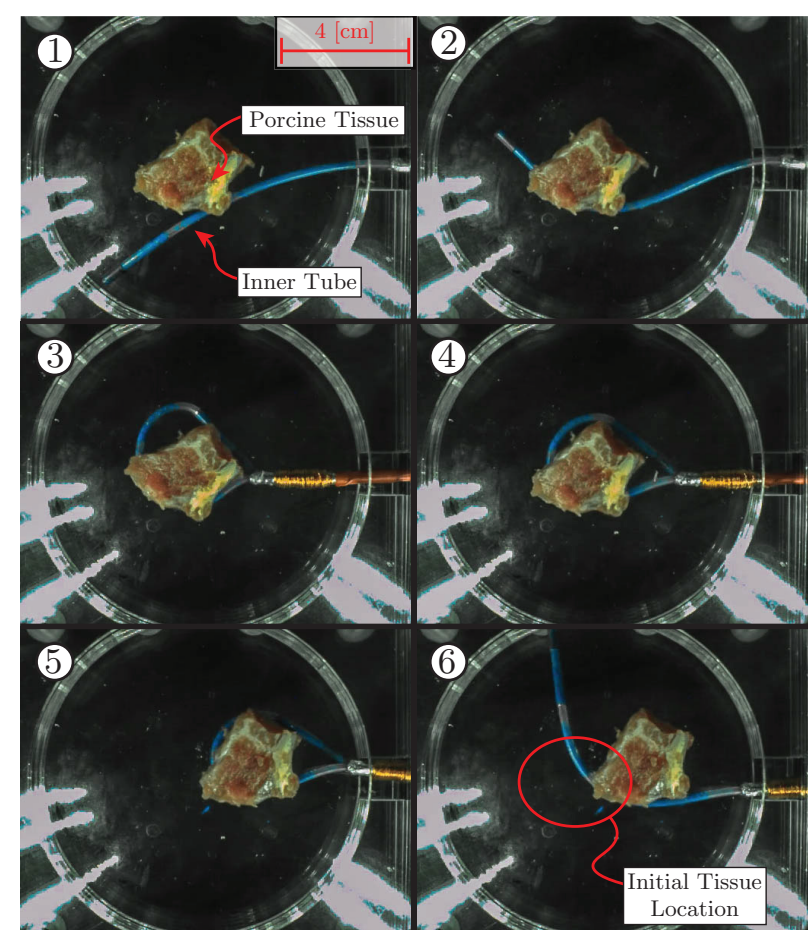

Fig. 8. The final experiment involving execution of a pick-and-place task using tentacle catheter. The catheter is used to grasp a $2 \times 2 \times 2 \mathrm{~cm}$ piece of porcine tissue located inside the workspace of BigMag array. Subsequently, the BigMag inserter has been used to move the object by retracting the catheter. After reaching the desired location, the current in the coil of tentacle catheter has been reversed to release the grasp. Please refer to the video in the supplementary material for demonstration of this experiment.

\section{Pick-and-Place Task}

The final proof-of-concept experiment employs the tentacle catheter for manipulation of porcine tissue (Figure 8). It serves to demonstrate that the grasping strategy presented in Figure 3 is applicable to pick-and-place tasks involving biological material. The tentacle catheter is operated at 0.75 A, as this current has been established safe by thermal study. The $2 \times 2 \times 2 \mathrm{~cm}$ sample of pork tissue is placed inside the workspace of BigMag. The mass of the sample is $9.5 \mathrm{~g}$. The tentacle catheter is wrapped around the tissue, the coil is activated to form a stable loop around it. Subsequently, the inserter of BigMag is used to retract the catheter, displacing the tissue. During the motion, reliable grasp has been observed. After the motion is finished, the current in the coil is reversed to release the catheter, which is later fully retracted from the workspace.

\section{COnClusions And Future Work}

In this work we present a proof-of-concept study of a magnetically-actuated tentacle catheter for grasping tasks within human body. The tentacle catheter is selected out of three concepts, based on its adherence to a set of design requirements. The prototype of the catheter is tested within the BigMag coil array. Since the heat dissipated by the catheter could potentially damage biological tissue, we test the safety of the catheter in a thermal study. Moreover, we show that using our proposed grasping technique, forces of up to $0.1 \mathrm{~N}$ can be applied for object manipulation. This force is in agreement with theoretical predictions. This force allows for manipulation of clinially-relevant samples of tissues.
Finally, we test the catheter by successfully accomplishing a pick-and-place task using porcine tissue.

In our future work, we plan to derive a mathematical model of under-actuated grasping using tentacle catheter. The model will be used in development of a control algorithm for automated manipulation of objects in confined environments using the tentacle catheter. We will validate the controller with more advanced pick-and-place experiments with samples of biological tissue of various sizes. Finally, we will test the closed-loop control of the catheter using clinicallyrelevant sensing modalities, such as ultrasound imaging and Fiber Bragg Grating sensors.

\section{REFERENCES}

[1] J. Burgner-Kahrs, D. C. Rucker, and H. Choset, "Continuum Robots for Medical Applications: A Survey," IEEE Transactions on Robotics, vol. 31 , no. 6 , pp. 1261-1280, 2015 .

[2] A. Ali, D. H. Plettenburg, and P. Breedveld, "Steerable Catheters in Cardiology: Classifying Steerability and Assessing Future Chal lenges," IEEE Transactions on Biomedical Engineering, vol. 63, no. 4, pp. 679-693, 2016

[3] G. J. Vrooijink, T. Ellenbroek, P. Breedveld, J. G. Grandjean, and S. Misra, "A preliminary study on using a Robotically-Actuated Delivery Sheath (RADS) for transapical aortic valve implantation,' Proceedings of 2014 IEEE International Conference on Robotics and Automation (ICRA), Hong Kong, China, pp. 4380-4386, 2014

[4] T. Kimura, S. Takatsuki, A. Oishi, M. Negishi, S. Kashimura, Y. Katsumata, T. Nishiyama, N. Nishiyama, Y. Tanimoto, and Y. Aizawa, "Operator-blinded contact force monitoring during pulmonary vein isolation using conventional and steerable sheaths," International journal of cardiology, vol. 177, no. 3, pp. 970-976, 2014.

[5] C. Majidi, "Soft robotics: a perspective - current trends and prospects for the future," Soft Robotics, vol. 1, no. 1, pp. 5-11, 2014

[6] C. M. Heunis, J. Sikorski, and S. Misra, "Magnetic actuation of flexible surgical instruments for endovascular interventions," IEEE Robotics and Automation Magazine, Accepted.

[7] J. Edelmann, A. J. Petruska, and B. J. Nelson, "Magnetic control of continuum devices," Int. J. Rob. Res., vol. 36, no. 1, pp. 68-85, 2017.

[8] V. N. T. Le, N. H. Nguyen, K. Alameh, R. Weerasooriya, and P. Pratten, "Accurate modeling and positioning of a magnetically controlled catheter tip," Medical Physics, vol. 43, no. 2, pp. 650-663, 2016.

[9] C. Chautems and B. J. Nelson, "The tethered magnet: Force and 5-dof pose control for cardiac ablation," in Proceedings of 2017 IEEE International Conference on Robotics and Automation (ICRA), Singapore. IEEE, 2017, pp. 4837-4842.

[10] A. D. Losey, P. Lillaney, A. J. Martin, D. L. Cooke, M. W. Wilson, B. R. H. Thorne, R. S. Sincic, R. L. Arenson, M. Saeed, and S. W. Hetts, "Magnetically assisted remote-controlled endovascular catheter for interventional MR imaging: in vitro navigation at $1.5 \mathrm{~T}$ versus X-ray fluoroscopy," Radiology, vol. 271, no. 3, pp. 862-869, 2014.

[11] S. L. Charreyron, B. Zeydan, and B. J. Nelson, "Shared control of a magnetic microcatheter for vitreoretinal targeted drug delivery," in Proceedings of 2017 IEEE International Conference on Robotics and Automation (ICRA), Singapore. IEEE, 2017, pp. 4843-4848.

[12] F. Ullrich, S. Schuerle, R. Pieters, A. Dishy, S. Michels, and B. J. Nelson, "Automated capsulorhexis based on a hybrid magneticmechanical actuation system," Proceedings of 2014 IEEE International Conference on Robotics and Automation (ICRA), Hong Kong, China, pp. 4387-4392, 2014.

[13] G. Dogangil, B. L. Davies, and F. R. Y Baena, "A review of medical robotics for minimally invasive soft tissue surgery," Proceedings of the Institution of Mechanical Engineers, Part H: Journal of Engineering in Medicine, vol. 224, no. 5, pp. 653-679, 2010.

[14] C. Di Natali, J. Buzzi, N. Garbin, M. Beccani, and P. Valdastri, "Closed-loop control of local magnetic actuation for robotic surgical instruments," IEEE Transactions on Robotics, vol. 31, no. 1, pp. 143 156, 2015.

[15] N. Giri and I. Walker, "Continuum robots and underactuated grasping," Mechanical Sciences, vol. 2, no. 1, pp. 51-58, 2011.

[16] S. W. Hetts, M. Saeed, A. J. Martin, L. Evans, A. F. Bernhardt V. Malba, F. Settecase, L. Do, E. J. Yee, and A. Losey, "Endovascular catheter for magnetic navigation under MR imaging guidance: Evaluation of safety in vivo at 1.5 T," American Journal of Neuroradiology, vol. 34, no. 11, pp. 2083-2091, 2013

[17] J. Sikorski, I. R. Dawson, A. Denasi, E. E. G. Hekman, and S. Misra "Introducing BigMag - a novel system for 3D actuation of flexible surgical devices," Proceedings of 2017 IEEE International Conference on Robotics and Automation (ICRA), Singapore, pp. 3594-3599, 2017.

[18] F. Burbank, S. H. Parker, and T. J. Fogarty, "Stereotactic breast biopsy: improved tissue harvesting with the mammotome." The American Surgeon, vol. 62, no. 9, pp. 738-744, 1996. 